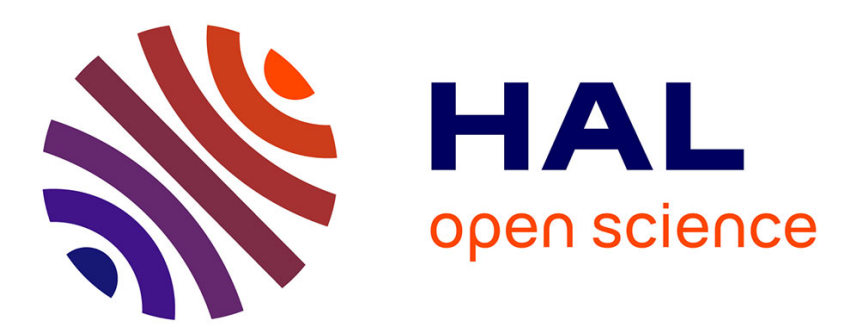

\title{
Le face-à-face OGM - agriculture biologique en Europe: entre réglementation et recommandation
}

Christophe Charlier, Alexandra Rufini

\section{To cite this version:}

Christophe Charlier, Alexandra Rufini. Le face-à-face OGM - agriculture biologique en Europe: entre réglementation et recommandation. Revue d'Economie Politique, 2013, 123 (4), pp.573-592. hal00872184

\section{HAL Id: hal-00872184 \\ https://hal.science/hal-00872184}

Submitted on 11 Oct 2013

HAL is a multi-disciplinary open access archive for the deposit and dissemination of scientific research documents, whether they are published or not. The documents may come from teaching and research institutions in France or abroad, or from public or private research centers.
L'archive ouverte pluridisciplinaire HAL, est destinée au dépôt et à la diffusion de documents scientifiques de niveau recherche, publiés ou non, émanant des établissements d'enseignement et de recherche français ou étrangers, des laboratoires publics ou privés. 


\section{Le face-à-face OGM - agriculture biologique en Europe : entre réglementation et recommandation}

Christophe CHARLIER* Maître de Conférences charlier@gredeg.cnrs.fr Tel : 04.93.95.43.41 (auteur correspondant)
Alexandra RUFINI* Maître de Conférences rufini@gredeg.cnrs.fr Tel : 04.93.95.42.45

16 mai 2013

*Université Nice Sophia-Antipolis - GREDEG/CnRS Adresse : 250 rue Albert Einstein, 06560 Valbonne, FRANCE 


\title{
Résumé
}

Le Règlement Européen 834/2007, en charge de définir le label Biologique européen depuis janvier 2009, autorise un seuil de $0.9 \%$ de présence fortuite d'OGM dans les produits Biologiques (Bio). Un seuil d'OGM différent de zero permet aux agriculteurs de préserver leur label dans le cas d'une faible contamination OGM mais risque de discréditer une filière de réputation "sans OGM". Dans la Recommandation 2003/556/CE la Commission européenne propose de faire supporter aux agriculteurs d'OGM les mesures de protection visant à limiter la contamination OGM -principe dit du newcomer- mais laisse le choix aux Etats membres d'appliquer ce principe. Ce papier présente une analyse économique des conséquences de ce seuil d'OGM sur le bien-être de la société avec et sans le principe du newcomer. Il étudie comment le régulateur impartial, face à des consommateurs hétérogènes, détermine le seuil d'OGM autorisé pour assurer la coexistence des deux formes d'agriculture, en tenant compte du discrédit mais aussi de la flexibilité qu'il engendre pour le maintien du label Bio. Après avoir défini les conditions de la coexistence des agricultures, nous montrons que i) le seuil d'OGM devrait être différent de zero lorsque les agriculteurs Bio supportent les mesures de coexistence et que ii) le seuil d'OGM autorisé devrait être nul lorsque le principe du newcomer est retenu dans la réglementation.

Codes JEL : K29, Q18, Q58

Mots clés : Agriculture Biologique, OGM, Réglementation.

\section{The confrontation of the GM and Organic agricultures in Europe : Between regulation and recommendation}

\begin{abstract}
The European Regulation 834/2007, responsible for the definition of the European Organic Label since 2009, allows a non-intentional presence of authorised GMOs limited to the threshold of $0.9 \%$ in organic production. Such a threshold, when strictly positive, allows organic producers to keep the organic label in case of a small GM contamination. However, it may bring discredit on the organic agriculture which can no more be understood as "GMO-free". The European Commission in its recommendation 2003/556/CE proposes that GM producers should bear the cost of the measures decided to limit the dissemination of the GM gene - the so-called "newcomer principle". European countries are however free to adopt such a principle and can proceed differently. This article presents an economic analysis of the determination of the optimal GMO threshold maximizing society's wellbeing when the newcomer principle is implemented and when it is not. This optimal GMO threshold in both cases is decided by an impartial regulator facing heterogeneous consumers, and is intended to ensure the coexistence between GM and organic agricultures, taking into account of the potential discredit it implies on organic agriculture and the flexibility it allows for the preservation of the organic label. We first establish the conditions for coexistence. We then show that i) the GMO threshold can be different from zero if the organic producers bear the cost of the coexistence measures and ii) the GMO threshold should be zero under the newcomer principle.
\end{abstract}


JEL Codes : K29, Q18, Q58

Keywords : Organic production, GMO, Regulation. 


\section{Introduction}

Le Règlement européen n ${ }^{\circ}$ 834/2007 sur l'agriculture biologique en vigueur depuis janvier 2009, fait suite au règlement 2092/91 pour harmoniser les normes en matière d'agriculture biologique avec l'adoption d'un cahier des charges commun pour la production biologique. Ce type d'agriculture a en effet été au cours du temps un réceptacle d'aspirations différentes allant du respect de l'environnement, à l'opposition à l' "agribusiness" en passant par l'attention portée à la santé humaine, au bien-être animal et à l'intégrité du vivant. Il est difficile d'intégrer tous ces éléments dans un label unique qui ne peut dès lors que réduire à certaines dimensions le sens donné à l'appellation "Agriculture Biologique". Le règlement européen a dû donc faire des choix sur le sens à donner à cette agriculture et sur les modalités techniques permettant de la mettre en œuvre.

Le label Bio actuel signifie que les produits qui le portent sont issus de processus de production qui n'ont pas recours à des éléments chimiques de synthèse, dans une limite de $5 \%$ des ingrédients. A l'utilisation d'engrais et de pesticides issus de l'industrie chimique seront ainsi préférés le recours aux engrais verts, l'introduction d'ennemis naturels des parasites, la rotation des cultures etc. Plus que la qualité intrinsèque des produits agricoles concernés, c'est le respect de l'environnement qui est ainsi visé par ce label ${ }^{1}$. Dans cette perspective environnementale, mais aussi pour ne pas courir de risques inutiles, l'objectif "non OGM" apparaissait au cahier des charges du label Bio français. Si l'interdiction d'utilisation des OGM dans la production biologique demeure, le cahier des charges du label Bio européen autorise un seuil de 0,9\% de présence accidentelle d'OGM. Ce seuil de tolérance OGM est identique à celui qui distingue les produits OGM des produits non OGM (produits dits "conventionnels"). Ainsi, la définition que retient le règlement européen pour le seuil de 0,9\% de présence OGM au-delà duquel un produit doit être étiqueté OGM est celle fixée par le règlement 1829/2003 à son article 12 para 2.

Dans l'esprit de la Commission Européenne qui a promu le règlement sur l'agriculture biologique, cette norme empêche une présence supérieure d'OGM dans les produits Bio et ne remet pas en cause l'interdiction de recourir intentionnellement aux OGM dans l'agriculture biologique. Les réactions des ONG environnementales ont cependant pointé, dès l'annonce du règlement européen en 2007, le discrédit qu'une telle norme risquait de porter à la filière Bio. Le débat est d'autant plus sensible que les OGM et l'agriculture Biologique représentent deux types de production agricole (voire d'innovation) en expansion que l'on oppose souvent ${ }^{2}$. La possibilité prévue par le règlement européen d'introduire 5\% d'ingrédients non Bio dans un aliment étiqueté Bio a comparativement moins cristallisé les opinions.

L'agriculture biologique et les OGM ont chacun fait l'objet de nombreuses études dans la littérature économique, même si leur confrontation n'a que peu été étudiée. Dans ces études l'accent a souvent été mis sur l'impossibilité pour le consommateur de vérifier les caractéristiques du système de production agricole d'où provient l'aliment qu'il consomme. Le label Bio (Giannakas 2002) comme l'étiquetage OGM (Fulton et Giannakas 2004) ont été alors considérés comme source d'information pour les consommateurs

1. Voir www.agence-bio.org.

2. Dans les pays comme les États-Unis où les OGM ne sont pas étiquetés, ce débat aurait un autre sens, l'étiquetage "Bio" permettant aux consommateurs de choisir de ne pas consommer des OGM. Voir Giannakas et Yiannakas (2006). 
venant corriger une asymétrie d'information. Les différentes formes d'étiquetage OGM ("contient", "ne contient pas", "peut contenir") ont ainsi été étudiées (Crespi et Marette 2003, Runge et Jackson 2000). Enfin, la valeur attribuée au label Bio (Batte et al. 2007, Nimon et Beghin 1999), la coexistence de plusieurs labels Bio et les effets sur le bien-être des consommateurs de leur harmonisation internationale (Sawyer et al. 2008) ont aussi fait l'objet d'études économiques. Dans ces travaux, le label Bio et/ou l'étiquetage des OGM sont étudiés comme des vecteurs d'information pour des biens agricoles dits de "confiance" ${ }^{3}$. Les situations avant et après étiquetage y sont ainsi analysées sous l'angle du bien-être de consommateurs hétérogènes.

La référence à un seuil de présence accidentelle d'OGM permet de déterminer ce qu'est un produit OGM et ce qui ne l'est pas. Le respect de ce seuil nécessite que la coexistence des agricultures biologique et OGM soit organisée. Cette question de la coexistence est initialement une problématique agronomique qui a rapidement eu des ramifications pluridisciplinaires ${ }^{4}$. En économie, l'évaluation de la propension à payer des consommateurs pour des aliments OGM $^{5}$ et l'évaluation du coût de la séparation des filières ont en particulier été explorées ${ }^{6}$.

Le fait que le seuil de présence accidentelle d'OGM soit différent de zéro, nous indique que le législateur européen prend en compte le coût de la coexistence et recherche sa faisabilité économique. Cette référence n'est cependant pas neutre. Définir un seuil de tolérance désigne implicitement l'agriculture OGM comme l'élément perturbateur. Cette désignation n'est toutefois explicite qu'à partir du moment où la réglementation rend les producteurs d'OGM responsables et demande que les mesures de coexistence soient à leur charge (ce que la littérature a appelé le "principe du newcomer") ${ }^{7}$. La littérature économique sur la coexistence s'est principalement intéressée à ce point en distinguant (Beckmann et al. 2006) les mesures de coexistence ex ante (distance entre les champs par exemple), des mesures de coexistence ex post (régime de responsabilité en cas de dissémination du gène OGM). Si l'efficacité de la combinaison de ces mesures a pu être étudiée (Beckmann et al. 2010), c'est principalement le bienfondé, d'un point de vue économique, de la décision réglementaire de mettre les mesures de coexistence à charge des producteurs d'OGM qui est discuté (Beckmann et al. 2010, Demont et al. 2009, Desquilbet et Bullock 2010, Gray et al. 2011, et Cedia et al. 2011). En considérant la dissémination du gène OGM comme une pollution spatiale, ces études se rejoignent généralement pour mettre en avant, sur la toile de fond du théorème de Coase, l'intérêt de permettre/encourager la négociation entre agriculteurs voisins de la taille des mesures

3. Biens dont les caractéristiques des processus de production ne peuvent pas être observées ou découvertes lors de la consommation. Voir les articles fondateurs de Nelson (1970) et Darby et Karni (1973). Dans ce contexte, le label Bio est censé mettre fin à une asymétrie d'information entre producteurs et consommateurs. Dans une situation plus compliquée, où l'incertitude serait partagée entre consommateurs, producteurs et experts (cas des biens "indéterminés" présenté par Lupton 2005), le problème de confiance se déporterait sur le label-même (Balindeau et Dufeu 2010).

4. Voir le programme de recherche européen Co-Extra (www.coextra.eu).

5. La littérature est ici très abondante. Pour une approche utilisant l'économie expérimentale, voir Noussair et al. (2004).

6. Voir par exemple Huygen et al. (2004).

7. Ce principe est issue d'une Recommandation 2003/556/CE de la Commission Européenne qui l'énonce comme suit : "En règle générale, lors de la phase d'introduction d'un nouveau type de production dans une région, les opérateurs (agriculteurs) qui en prennent l'initiative devraient s'engager à mettre en oeuvre les mesures de gestion agricoles nécessaires à la limitation du flux génétique". 
de coexistence, de leur localisation géographique et de leur prise en charge financière. En outre, les conséquences de la règlementation sur la localisation des activités (intérêt et incitations à la constitution de zones franches d'OGM et de zones OGM) sont étudiées.

La question que traite cet article se situe en amont de ces travaux sur la coexistence. Nous montrons que la répartition de la charge du coût de la coexistence (i.e. l'adoption ou non du principe du newcomer) n'est pas neutre à l'égard du seuil de présence accidentelle d'OGM lorsque la définition de celui-ci répond à un critère d'optimalité (maximisation du bien-être de la société). Ainsi, ce seuil de tolérance OGM peut être strictement positif lorsque le principe du newcomer n'est pas retenu, alors qu'il est nul dès que ce principe est reconnu. Pour établir ces résultats nous reprenons le contexte d'hétérogénéité des préférences des consommateurs retenu généralement dans la littérature sur les labels. Cette hétérogénéité des préférences nous permet de tenir compte du discrédit qu'engendre l'autorisation d'une présence accidentelle limitée d'OGM pour l'agriculture biologique. Nous considérons en outre que les consommateurs sont parfaitement informés par le label agriculture biologique et l'étiquetage OGM réglementaires ${ }^{8}$.

L'article est organisé de la manière suivante. Le modèle est présenté et la condition sous laquelle apparaît la coexistence des deux agricultures biologique et OGM est établie dans la section 2. Le seuil optimal de présence OGM accidentelle lorsque le principe du newcomer n'est pas retenu dans la réglementation (et donc que le coût des mesures de coexistence est à charge des producteurs Bio) est déterminé dans la section 3. La situation où le principe du newcomer est retenu est traitée à la section 4 . La dernière section conclut.

\section{Le modèle}

Une denrée agricole peut être produite de deux manières différentes, en ayant recours aux biotechnologies $g m$ ou à l'agriculture biologique $o$, et est vendue sur deux marchés différents (supposés concurrentiels) à deux prix différents : $p_{g m}$ et $p_{o}$. Un règlement qui doit être respecté par les producteurs Bio fixe $l \in[0 ; 1]$, un seuil maximal de présence OGM accidentelle $^{9}$. Si ce seuil de tolérance est dépassé, la production est étiquetée OGM et vendue sur le marché OGM, ce qui représente une perte pour le producteur Bio dans la mesure où $p_{g m}$ est toujours inférieur à $p_{o}{ }^{10}$.

\subsection{Les producteurs}

La différence des technologies est formellement représentée avec des fonctions de coût de production différentes. Nous supposons que la production OGM a la fonction de coût total suivante :

$$
C_{g m}\left[q_{g m}\right]=c_{g m} q_{g m}
$$

8. Cette hypothèse est simplificatrice. Même s'ils observent le label, les consommateurs peuvent ne pas pleinement le comprendre, en particulier si son cahier des charges est compliqué. Le problème de la confiance à l'égard du label peut en outre se poser (voir note de bas de page 3 ).

9. Dans la pratique, une présence d'OGM inférieure à $0.01 \%$ n'est pas détectable techniquement.

10. C'est la raison pour laquelle nous ne considérons pas dans ce papier les produits issus de l'agriculture conventionnelle, c'est-à-dire qui ne sont ni Bio ni OGM. 
Où $q_{g m}$ est la quantité d'OGM produite et $c_{g m}$ est le coût marginal constant ${ }^{11}$.

L'agriculture biologique a pour fonction de coût :

$$
C_{o}\left[q_{o}\right]=\left(c_{o}+S[l]\right) q_{o}
$$

Où $q_{o}$ est la quantité de produits Bio offerte et $c_{o}$ la partie technologique du coût marginal. La fonction $S[l]$ représente l'impact sur le coût marginal de l'agriculture biologique du contrôle ex ante de la pollution OGM nécessaire pour respecter le seuil d'OGM accepté dans la réglementation $l$. Ce coût est en particulier engendré par l'utilisation de matériel spécifique (ou nettoyé de toute trace d'OGM), l'attention portée à la pureté des semences, et le maintien d'un espace entre les cultures de types différents. L'hypothèse suivante est posée sur les dérivées de la fonction $S[l]$ :

Hypothèse 1. Plus le seuil de tolérance est élevé et moins la pollution OGM est coûteuse pour un producteur Bio (il est moins coûteux pour le producteur d'avoir la certification "Bio") et ce, à taux décroissant : $\partial S / \partial l<0$ et $\partial^{2} S / \partial l^{2}>0$.

Cette hypothèse est importante car dans le débat qui a accompagné la réglementation européenne elle constituait un des arguments des défenseurs de l'introduction d'un seuil de présence accidentelle OGM dans l'agriculture biologique. Le seuil était ici défendu car, en rendant une pollution OGM moins dommageable, il permettait une flexibilité dans la gestion du label Bio.

En outre, nous posons deux conditions sur les bornes de la fonction $S[l]$ avec l'hypothèse suivante :

Hypothèse 2. Si la réglementation impose une absence totale d'OGM dans l'agriculture biologique, alors le coût de sa mise en ouvre est maximal : $S[l=0]=S_{\max }$. A l'inverse, si la réglementation est du type "une production Bio peut être OGM", le coût du respect du label du point de vue de la présence OGM est nul : $S[l=1]=0$.

Les deux situations qui apparaissent ainsi dans l'Hypothèse 2 doivent être entendues comme des cas polaires. Elles sont peu réalistes pour des raisons de natures différentes (technique pour la première et politique pour la seconde). Elle bornent cependant l'ensemble des valeurs de $l$.

Nous utiliserons, lorsque nécessaire, la spécification suivante pour la fonction $S[l]$ : $S[l]=\lambda(1-l)^{2}$ où $\lambda$ est un paramètre strictement positif mesurant l'importance de la difficulté du contrôle de la pollution OGM.

Le coût marginal de la production Bio $c_{o}+S[l]$ est supposé supérieur au coût marginal $c_{g m}$ des producteurs d'OGM du fait de la pollution OGM à contrôler et du coût engendré par le règlement sur l'agriculture biologique (cahier des charges à respecter).

\subsection{Les consommateurs}

Nous considérons un marché avec $n$ consommateurs hétérogènes quant à leurs préférences sur le respect de l'environnement des processus de production dont sont issus les

11. Cette hypothèse de coût marginal constant est simplificatrice. Nous supposons que les producteurs ont une quantité de production maximale qui est effectivement produite dès que le prix de l'output est égal au coût marginal. 
biens qu'ils consomment. La préférence environnementale d'un consommateur est notée $e$. Nous supposons que les consommateurs sont uniformément répartis sur le segment $[0,1]$. Si $e=0$, le consommateur n'attribue aucune importance à l'environnement. Si, au contraire, $e=1$, il attribue la plus grande importance envisageable à l'environnement.

Les fonctions individuelles de demande d'OGM sont supposées linéaires du prix et sont paramétrées par la préférence environnementale du consommateur $i$ :

$$
d_{g m}[e]=a_{g m}[e]-b_{g m} p_{g m}
$$

Où $b_{g m}$ est un paramètre mesurant la réactivité de la demande d'OGM par rapport au prix des OGM et où $a_{g m}[e]$ est la quantité maximale de produits OGM demandée, telle que $a_{g m}[e] \geq 0$ et $a_{g m}{ }^{\prime}[e]<0$.

Les fonctions de demande individuelles de produits Bio sont elles aussi supposées linéaires du prix, tout en étant paramétrées par la préférence environnementale du consommateur et le seuil de tolérance OGM ${ }^{12}$ :

$$
d_{o}[e]=a_{o}[e, l]-b_{o} p_{o}
$$

Où $b_{o}$ est un paramètre mesurant la réactivité de la demande de produit Bio par rapport à son prix (notons que par souci de réalisme, on aura $b_{g m}>b_{o}$ dans la mesure où les produits OGM sont plus sensibles au prix que les produits Bio) et où $a_{o}[e, l]$ est la quantité maximale de produits Bio demandée, telle que $a_{o}[e, l] \geq 0$ et $a_{o}{ }^{\prime}[e]>0$. Nous supposons que, $\forall e$, cette demande maximale $a_{o}[e, l]$ est supérieure à celle des OGM $a_{g m}[e]$ afin de tenir compte de la différenciation verticale des produits. En effet, si les prix sont identiques, le produit Bio est considéré de meilleure qualité, ce qui engendre une demande plus élevée.

Nous posons par ailleurs l'hypothèse suivante qui nous permet de rendre compte du discrédit qu'un seuil de présence OGM autorisé différent de zéro engendre sur l'agriculture biologique :

Hypothèse 3. Plus le seuil autorisé d'OGM dans les produits Bio est grand, et plus l'agriculture biologique est banalisée sur le plan de son contenu OGM, et donc plus la quantité maximale de produits Bio demandée est faible : $a_{o}{ }^{\prime}[l]<0$

Lorsque nécessaire, nous utiliserons les spécifications les plus simples possible pour chacune des deux fonctions de demande, telles que : $d_{g m}[e]=\bar{a}_{g m}(\alpha-e)-b_{g m} p_{g m}$ et $d_{o}[e]=\bar{a}_{o}(\alpha+e-l)-b_{o} p_{o}$ où $\alpha$ est un paramètre tel que $\alpha>1$ et où $\bar{a}_{g m}$ et $\bar{a}_{o}$ sont des paramètres de demande strictement positifs.

Compte tenu des fonctions de demande décrites par les équations (3) et (4), nous pouvons écrire les surplus nets des consommateurs pour les deux types de consommation tels que :

$$
\operatorname{Surplus}_{g m}\left[e, p_{g m}\right]=\frac{\left(a_{g m}[e]-b_{g m} p_{g m}\right)^{2}}{2 b_{g m}}
$$

12. Ce seuil n'est pas un paramètre des fonctions de demande d'OGM (3) car nous supposons qu'un consommateur qui décide d'acheter des produits OGM n'accorde pas d'importance particulière au seuil de tolérance OGM dans l'agriculture biologique. 


$$
\operatorname{Surplus}_{o}\left[e, l, p_{o}\right]=\frac{\left(a_{o}[e, l]-b_{o} p_{o}\right)^{2}}{2 b_{o}}
$$

Ainsi, conformément à l'intuition, le surplus net d'un consommateur d'OGM décroît avec sa sensibilité environnementale. Inversement, le surplus net d'un consommateur de produits Bio augmente avec sa sensibilité à l'environnement tandis qu'il décroît avec le seuil autorisé de présence OGM dans les produits Bio.

\subsection{La coexistence}

Nous définissons la coexistence de l'agriculture biologique et de l'agriculture OGM au sens économique de la manière suivante. La coexistence de l'agriculture biologique et de l'agriculture OGM est réalisée, lorsque des échanges sur les deux marchés correspondants existent. En d'autres termes, il est nécessaire que nous trouvions des consommateurs et des producteurs sur les deux marchés du produit Bio et du produit OGM ${ }^{13}$. La condition de la coexistence est donnée dans la Définition 1.

Définition 1. La coexistence de l'agriculture biologique et de l'agriculture OGM est assurée si :

a) $p_{g m} \geq c_{g m}$

b) $p_{o} \geq c_{o}+S[l]$

c) Pour $p_{o}, p_{g m}$ et $l$ donnés, il existe un consommateur ẽ indifférent entre consommer des produits Bio et des produits OGM, i.e., ẽ tel que $\operatorname{Surplus}_{o}\left(e, l, p_{o}\right)=\operatorname{Surplus}_{g m}\left(e, p_{g m}\right)$ et $\tilde{e} \in] 0 ; 1[$.

L'interprétation de la Définition 1 est la suivante. La coexistence est assurée si une offre et une demande existe pour chacun des deux produits agricoles. Pour cela, les prix sur chacun des marchés doivent être tels que les profits des producteurs soient positifs ou nuls (conditions $a$ et $b$ ). La condition $b$ nous montre en outre que le prix du produit Bio doit être suffisamment élevé (supérieur d'un montant de $S[l]$ à la partie technique du coût marginal $c_{o}$ ) pour qu'une offre puisse apparaître. Par ailleurs, la coexistence est réalisée si les surplus des consommateurs lorsqu'ils consomment l'un des deux biens ne sont pas systématiquement supérieurs aux surplus lorsqu'ils consomment l'autre bien. En d'autres termes, il est nécessaire qu'il existe un consommateur indifférent entre les deux biens (condition $c$ ).

En définissant un seuil de présence OGM accidentelle autorisée différent de zéro dans les produits Bio, le régulateur européen reconnaît implicitement qu'une pollution OGM ne peut pas être évitée ou ne pourrait l'être qu'à un coût important. La possibilité d'une coexistence organisée par ce seuil de tolérance OGM est ainsi souvent avancée comme un résultat positif pour la raison de non exclusion a priori de certaines formes d'agriculture

13. La définition de la coexistence donnée ici, faisant référence aux marchés, est de nature économique. La coexistence a cependant fait principalement l'objet de travaux agronomiques. Voir www.coextra.eu. Du point de vue juridique, la coexistence permet la liberté de choix des consommateurs (consommer ou pas des OGM), comme la liberté de choix des producteurs (produire ou pas des OGM). 
voire de la protection de l'environnement ${ }^{14}$. Dans le débat qui a accompagné le Règlement européen ce seuil était ainsi défendu comme un élément permettant aux producteurs Bio de ne pas perdre le label "Agriculture Biologique" en cas de pollution OGM contenue en-dessous de ce seuil. A quel niveau, dès lors, le régulateur devrait-il fixer le seuil de tolérance OGM dans l'agriculture Biologique? Cette question est traitée dans la section qui suit en considérant non seulement la situation des producteurs mais aussi celle des consommateurs pour lesquels ce seuil de tolérance est important.

\section{Le seuil optimal de présence OGM accidentelle}

Pour déterminer le seuil de tolérance OGM optimal nous considérons que $l$ est une variable de contrôle pour un régulateur impartial qui chercherait à maximiser le surplus total de la société. Lorsque les coûts marginaux sont constants et les prix des deux produits Bio et OGM égaux à ceux-ci, les profits réalisés sont nuls et le surplus de la société $S T$ est égal à la somme des surplus des consommateurs.

$\mathrm{Au}$ prix $p_{o}=c_{o}+S[l]$ et $p_{g m}=c_{g m}$, le régulateur impartial cherche à résoudre le problème suivant :

$$
\max _{l \in[0,1]} S T[l]=n \int_{e=0}^{\tilde{e}[l]} \frac{\left(a_{g m}[e]-b_{g m} p_{g m}\right)^{2}}{2 b_{g m}} \mathrm{~d} e+n \int_{e=\tilde{e}[l]}^{1} \frac{\left(a_{o}[e, l]-b_{o} p_{o}\right)^{2}}{2 b_{o}} \mathrm{~d} e
$$

avec $\tilde{e}[l] \in] 0,1\left[\right.$ afin de ne considérer que les cas de coexistence des agricultures ${ }^{15}$.

Les premier et second termes de l'équation 7 représentent respectivement l'ensemble des agents qui choisissent de consommer des OGM et l'ensemble des agents qui choisissent de consommer des produits Bio. La FiguRE 1 représente cette distribution des consommateurs en fonction de leur sensibilité environnementale.

Proposition 1. (I) Si la coexistence des deux formes d'agriculture est assurée, il existe toujours un seuil optimal de présence OGM accidentelle autorisée dans l'agriculture $l^{*}$.

(II) Si les fonctions de demande sont linéaires en e, si $a_{o}{ }^{\prime \prime}[l, e]=0$ et $a_{o}^{\prime \prime}[l] \leq 0$, ce seuil optimal de présence OGM autorisée $l^{*}$ :

(i) est tel que les gains en flexibilité pour le producteur Bio $\left(b_{o} S^{\prime}[l]\right)$ égalisent les pertes liées au discrédit sur l'agriculture Bio ( $\left.a_{o}{ }^{\prime}[l]\right)$, c'est-à-dire $: b_{o} S^{\prime}[l]=a_{o}{ }^{\prime}[l]$.

(ii) est une solution intérieure au problème de maximisation si $b_{o} S^{\prime}[l=0]>a_{o}^{\prime}[l=0]$.

(iii) est tel qu'il maximise la part $(1-\tilde{e}[l])$ des consommateurs de produits Bio dans l'ensemble des consommateurs.

14. Ceci apparaît dans les textes législatifs européens. Voir Commission Recommendation of 23 July 2003 on guidelines for the development of national strategies and best practices to ensure the coexistence of genetically modified crops with conventional and organic farming, Official Journal, L 189, 29/07/2003, P. 0036 - 0047.

15. Néanmoins, notons que dans le cas plus général où la coexistence ne serait pas assurée, il faut remplacer $\tilde{e}[l]$ par sup[inf $[\tilde{e}[l], 1], 0]$ dans la fonction de surplus total. Le fait de considérer que la fonction $\tilde{e}[l]$ est strictement comprise entre 0 et 1 dans le surplus total exclut donc par définition les cas de non coexistence des agricultures sans pour autant limiter la généralité de notre modèle. En effet, borner la fonction $\tilde{e}[l]$ permet d'exclure les cas triviaux où i) il n'existe pas de marché du Bio (dans ce cas le seuil de pollution OGM n'existe plus) et ii) il n'existe pas de marché OGM (dans ce cas le problème de pollution OGM est évacué). 
Surplus

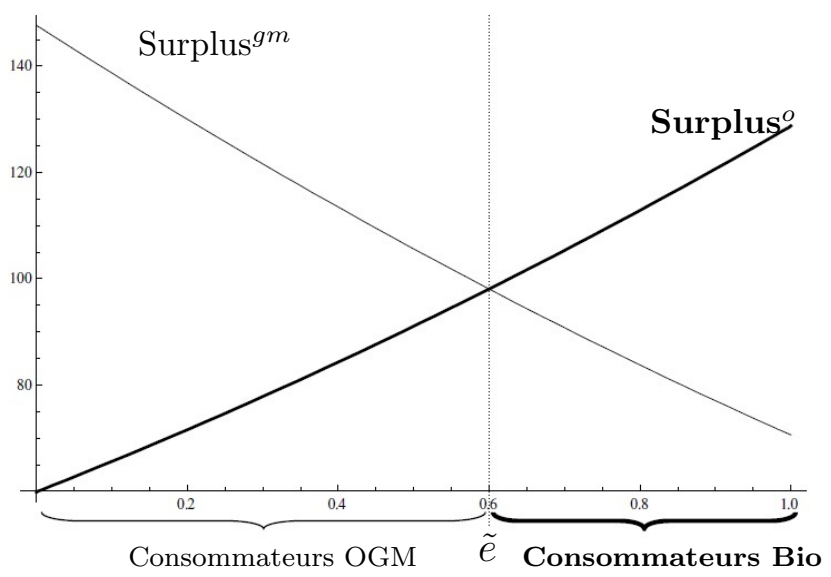

FiguRE 1 - Distribution des consommateurs en fonction de leur sensibilité à l'environnement dans un cas de coexistence des agricultures

Preuve. voir Annexe A.

Ainsi, la maximisation du bien être de la société consiste dans le même temps à maximiser le nombre possible de consommateurs Bio. Le choix de ce seuil optimal de présence OGM dépend des caractéristiques des demandes et des profits Bio et OGM. Compte tenu des conditions de positivité des demandes et de coexistence des agricultures il est possible d'écrire analytiquement le seuil optimal $l^{*}$ :

$$
l^{*}= \begin{cases}1-\frac{\bar{a}_{o}}{2 b_{o} \lambda} & \text { si } \bar{a}_{o}<2 b_{o} \lambda \\ 0 & \text { sinon }\end{cases}
$$

Avec l'équation 8 , on remarque que le régulateur social ne choisira jamais le cas très particulier où un produit Bio pourrait être OGM, i.e. $l^{*}=1^{16}$.

Par ailleurs, l'équation 8 nous permet de mettre en évidence les relations qui existent entre le seuil optimal et les paramètres du modèle. Nous pouvons distinguer deux types de paramètres : ceux qui influent seulement sur les conditions de positivité des demandes et/ou de coexistence des agricultures $\left(\bar{a}_{g m}, \alpha, c_{g m}, c_{o}, n\right)$ et ceux qui ont également une incidence sur le choix du seuil optimal $\left(\bar{a}_{o}, b_{o}, \lambda\right)^{17}$. En particulier, on remarque que plus l'envergure du coût de pollution OGM est importante (plus $\lambda$ est grand) et plus le seuil choisi par le régulateur sera proche de 1. Ceci traduit l'idée que plus le dommage d'une pollution OGM est grand et plus la flexibilité accordée par le seuil maximal de présence OGM devrait être grande. Cette caractéristique découle du fait qu'aucune autre forme de dédommagement des producteurs Bio qui perdraient leur label du fait d'une pollution OGM n'apparaît. Une "flexibilité totale" que permettrait un seuil de $100 \%$ ne sera pour autant jamais atteinte. Enfin, même s'il constitue un cas limite, un seuil nul ne doit pas être écarté. L'équation 8 nous montre qu'interdire toute trace d'OGM dans l'agriculture

16. En effet, $l^{*}=1$ si et seulement si $\frac{\bar{a}_{o}}{2 b_{o} \lambda} \leq 0$, ce qui est impossible puisque tous les paramètres sont positifs.

17. Avec l'équation 8 , on trouve : $l^{\prime}\left(\bar{a}_{o}\right)<0, l^{\prime}\left(b_{o}\right)>0$ et $l^{\prime}(\lambda)>0$. 
Bio est en effet optimal lorsque la "demande incompressible" de produits Bio, $\bar{a}_{o}$, est particulièrement élevée (supérieure à $\left.\overline{2} b_{o} \lambda\right)^{18}$.

\section{Le principe du newcomer}

Lorsque le principe du newcomer est retenu, les mesures de coexistence sont prises par les producteurs OGM. La fonction $S[l]$ représentant l'impact sur le coût marginal du contrôle de la pollution OGM nécessaire pour respecter le seuil d'OGM accepté dans la réglementation $l$ est donc à présent introduite dans la fonction de coût des producteurs OGM. Les fonctions de coûts sont alors modifiées comme suit :

$$
\begin{gathered}
C_{o}\left[q_{o}\right]=c_{o} q_{o} \\
C_{g m}\left[q_{g m}\right]=\left(c_{g m}+S[l]\right) q_{g m}
\end{gathered}
$$

L'hypothèse de décroissance à taux décroissant de la fonction $S[l]$ et les conditions aux bornes posées précédemment sont maintenues. En outre, nous supposons toujours que le coût marginal de la production Bio $c_{o}$ est supérieur au coût marginal $c_{g m}+S[l]$ des producteurs d'OGM du seul fait, à présent, du respect du cahier des charges de l'agriculture Biologique.

Examinons dans ces conditions le seuil de tolérance OGM optimal.

Au prix $p_{o}=c_{o}$ et $p_{g m}=c_{g m}+S[l]$, le régulateur impartial cherche désormais à résoudre le problème suivant :

$$
\max _{l \in[0,1]} S T^{N C}[l]=n \int_{e=0}^{\tilde{e}[l]} \frac{\left(a_{g m}[e]-b_{g m} p_{g m}\right)^{2}}{2 b_{g m}} \mathrm{~d} e+n \int_{e=\tilde{e}[l]}^{1} \frac{\left(a_{o}[e, l]-b_{o} p_{o}\right)^{2}}{2 b_{o}} \mathrm{~d} e
$$

Proposition 2. (I) Si la coexistence des deux formes d'agriculture est assurée, il existe toujours un seuil optimal de présence OGM accidentelle autorisée dans l'agriculture $l^{*}$.

(II) Si les fonctions de demande sont linéaires en e, si $a_{o}{ }^{\prime \prime}[l, e]=0$ et $a_{o}^{\prime \prime}[l] \leq 0$, ce seuil optimal de présence OGM autorisée $l^{*}$ :

(i) est une solution en coin au problème de maximisation, telle que $l^{*}=0$.

(ii) est tel qu'il maximise la part $(1-\tilde{e}[l])$ des consommateurs de produits Bio dans l'ensemble des consommateurs.

Nous montrons ainsi qu'en faisant supporter le coût des mesures de coexistence aux producteurs OGM plutôt qu'aux producteurs Bio, le seuil optimal de présence OGM autorisée se modifie. Avec le principe du newcomer, le régulateur devrait interdire toute pollution OGM dans les produits Bio. Dans cette configuration, les gains en flexibilité pour le producteur OGM $S^{\prime}[l]$ ne sont donc pas exploités.

Nous montrons également que, dans un contexte de différenciation verticale des produits, l'aversion pour les OGM conduit le régulateur à maximiser le nombre de consommateurs de produits Bio.

Ces deux résultats sont intimement liés. Si maximiser le surplus total revient à privilégier les consommateurs de produits Bio, et si le coût de la coexistence ne pèse pas

18. Dans ce cas, $S^{\prime}[l=0]<a_{o}^{\prime}[l=0]$, conformément à (II) (ii) : l'effet négatif du discrédit l'emporte sur l'effet positif de la flexibilité dès $l=0$. 
sur le prix des produits Bio mais sur celui des OGM, alors le surplus de la société est maximal lorsque les consommateurs Bio consomment un produit Bio "sans OGM".

La valeur nulle trouvée pour le seuil optimal de tolérance lorsque le principe du newcomer est introduit tient à certaines hypothèses posées dans notre modèle $\left(a_{o}[e, l]>a_{g m}[e]\right.$ et $b_{o}<b_{g m}$ en particulier). Cependant, plus que la valeur exacte de ce seuil, c'est l'idée que le principe du newcomer n'est pas neutre à l'égard de la détermination du seuil optimal de tolérance OGM qui est marquante. Le principe du newcomer désigne la responsabilité des producteurs d'OGM. Un des résultats importants de notre analyse est ainsi de montrer que les règles de responsabilité des producteurs d'OGM ne sont pas neutres à l'égard de la détermination du seuil de tolérance OGM. Ce résultat questionne le choix des autorités européennes d'avoir légiféré sur le seuil de tolérance OGM indépendamment de la fixation des règles sur la responsabilité des producteurs d'OGM. Si le seuil de tolérance est en effet précisé dans le règlement 1830/2003, il n'existe pas en droit européen de régime spécifique de responsabilité pour les producteurs d'OGM. L'initiative en la matière est laissée aux Etats Membres de l'Union Européenne. C'est ainsi que le principe du newcomer a simplement été inscrit dans une recommandation de la Commission Européenne ${ }^{19}$ et non dans un Règlement ou Directive. L'idée de faire porter la responsabilité de l'introduction des OGM aux producteurs d'OGM a cependant été largement reprise par les Etats Membres de l'Union Européenne. A titre d'exemple ${ }^{20}$, la France a instauré un régime de responsabilité respectant l'idée du principe du newcomer avec la loi du 25 juin 2008 relative aux organismes génétiquement modifiés.

\section{Conclusion}

En précisant le seuil de tolérance OGM dans un produit Bio, le règlement européen 834/2007 définit ce qu'est une pollution OGM dommageable pour un producteur Bio (celle qui engendre la perte du label Bio). Ce faisant, le règlement européen rompt l'image "sans OGM" attachée historiquement à l'agriculture biologique au risque de la discréditer. Nous cherchons à donner un "fondement économique" à ce type de réglementation en considérant que le seuil de tolérance OGM devrait être déterminé à partir de la maximisation du bien-être de la société. Sous les spécifications retenues dans le modèle développé dans cet article, les résultats obtenus nous montrent que le seuil de tolérance optimal peut ne pas être nul si les mesures de coexistence sont à charge des producteurs Bio. Le seuil de tolérance OGM optimal met en balance deux effets contradictoires sur le surplus des consommateurs Bio. Le premier effet est positif : si le seuil de tolérance diminue, les consommateurs Bio recherchant une consommation sans OGM voient leur surplus augmenter. Cependant, et c'est là la source du second effet, le contrôle de la pollution OGM nécessaire au maintien du label Bio devient plus difficile, de sorte que le prix du produit Bio augmente, pénalisant les consommateurs Bio. Dès que le principe du newcomer est adopté, et que la charge des mesures de coexistence est transférée aux producteurs OGM, le seuil de tolérance OGM optimal est nul. En effet, la maximisation du surplus total

19. Recommandation de la Commission Européenne 2003/556/CE "établissant des lignes directrices pour l'élaboration de stratégies nationales et de meilleures pratiques visant à assurer la coexistence des cultures génétiquement modifiées, conventionnelles et biologiques".

20. Voir Ngo (2011). 
conduit à maximiser le nombre de consommateurs Bio sensibles au seuil de tolérance OGM. Lorsque le coût des mesures de coexistence ne pèse plus sur le prix du produit Bio mais sur celui du produit OGM, le surplus total est maximum lorsque les consommateurs Bio consomment le produit Bio sans OGM.

Il est peu probable que l'impact du principe du newcomer sur le seuil de tolérance OGM à choisir ait été pris en compte dans le choix de la du Parlement européen et du Conseil de l'Union Européenne de fixer celui-ci à $0,9 \%$. Celui-ci a probablement été le fruit d'un processus de décision politique tenant compte des pressions des lobbies (Demont et Devos 2008). Le découplage du choix du seuil de tolérance OGM réglementaire et du principe du newcomer est d'ailleurs apparent. C'est en effet le règlement 1830/2003 dont l'application est obligatoire qui fixe ce seuil, alors que le principe du newcomer apparait dans la recommandation de la Commission Européenne 2003/556/CE dont l'application est laissée à la discrétion des États.

\section{Annexes}

\section{Annexe A : Démonstration de la Proposition 1}

\section{(I) Démonstration de l'existence de $l^{*}$}

Considérons l'expression du surplus total donnée par l'équation (7). Dans cette expression, comme $\tilde{e}[l]$ (qui est la solution de l'équation qui égalise les surplus individuels tout en respectant les conditions de positivité des demandes individuelles), $a_{o}[l]$ et $S[l]$ sont des fonctions continues par rapport à $l$, alors $S T[l]$ est également continue par rapport à $l$. Étant donné que la variable de contrôle $l$ est bornée entre 0 et 1 , la fonction de surplus total $S T[l]$ atteint, par conséquent, toujours au moins 1 maximum en $l$ sur l'intervalle $[0,1]$ et ce, quelles que soient les valeurs des paramètres qui assurent la coexistence des agricultures.

\section{(II) (i) Démonstration de la détermination de $l^{*}$ qui maximise le surplus total}

Afin de simplifier l'expression du surplus qui apparaît dans l'équation (7), nous posons que les fonctions de demande $a_{g m}[e]$ et $a_{o}[e, l]$ sont linéaires en $e$ et que la dérivée croisée de la demande Bio est nulle $a_{o(e, l)}^{\prime \prime}=0$. Ces simplifications assurent l'unicité du séparateur $\tilde{e}$ et nous permettent de réécrire le surplus tel que :

$$
S T[l]=n \int_{e=0}^{\tilde{e}[l]}(F-G e)^{2} \mathrm{~d} e+n \int_{e=\tilde{e}[l]}^{1}\left(a_{o}[l]+X e-b_{o} S[l]-Y\right)^{2} \mathrm{~d} e
$$

où : $F=a_{g m}-b_{g m} c_{g m} ; G e=a_{g m}[e]$ avec $F-G>0$ (condition de positivité de la demande OGM) ; Xe $=a_{o}[e] ; Y=b_{o} c_{o}$ avec $a_{o}[l]-b_{o} S[l]-Y>0$ (condition de positivité de la demande Bio).

La maximisation du surplus par rapport à $l$ conduit à poser la CPO suivante :

$$
\frac{d S T[l]}{d l}=0 \Rightarrow(1-\tilde{e})\left(2 a_{o}[l]+X \tilde{e}+X-2 b_{o} S[l]-2 Y\right)\left(a_{o}^{\prime}[l]-b_{o} S^{\prime}[l]\right)=0
$$

Ainsi, quelle que soit la valeur de $\tilde{e}$, le surplus total admet un extremum pour une valeur de $l^{*}$ telle que $a_{o}^{\prime}[l]=b_{o} S^{\prime}[l]$. 
L'étude de la CSO donne:

$\frac{d^{2} S T[l]}{d l^{2}}=(1-\tilde{e})\left(-2\left(a_{o}^{\prime}[l]-b_{o} S^{\prime}[l]\right)^{2}+\left(2 a_{o}[l]+X \tilde{e}+X-2 b_{o} S[l]-2 Y\right)\left(a_{o}^{\prime \prime}[l]-b_{o} S^{\prime \prime}[l]\right)\right)$

Comme $a_{o}^{\prime}[l]=b_{o} S^{\prime}[l]$, on peut écrire :

$$
\frac{d^{2} S T[l]}{d l^{2}}=(1-\tilde{e})\left(\left(2 a_{o}[l]+X \tilde{e}+X-2 b_{o} S[l]-2 Y\right)\left(a_{o}^{\prime \prime}[l]-b_{o} S^{\prime \prime}[l]\right)\right)<0
$$

Tous les facteurs de la CSO étant positifs sauf $a_{o}^{\prime \prime}[l]-b_{o} S^{\prime \prime}[l]$ dès lors que $a_{o}^{\prime \prime}[l] \leq 0$ (rappelons que nous avons posé $S^{\prime \prime}[l]>0$ ), on en déduit que $\frac{d^{2} S T[l]}{d l^{2}}<0$ et donc que la fonction de surplus total atteint bien un maximum pour une valeur de $l^{*}$ telle que $a_{o}^{\prime}[l]=b_{o} S^{\prime}[l]$.

Avec la spécification retenue, $a_{o}^{\prime}[l]=-\bar{a}_{o}$ et $b_{o} S^{\prime}[l]=2 b_{o} \lambda(l-1)$, on en déduit : $l^{*}=1-\frac{\bar{a}_{o}}{2 b_{o} \lambda}$.

(II) (ii) Démonstration de la détermination de $l^{*}$ en tant que solution intérieure au problème de maximisation

Étudions la dérivée de la fonction de surplus : si $b_{o} S^{\prime}[l]>a_{o}^{\prime}[l]$, alors $\frac{d S T[l]}{d l}>0$ et si $b_{o} S^{\prime}[l]<a_{o}^{\prime}[l]$, alors $\frac{d S T[l]}{d l}<0$. En termes économiques, tant que le risque de discrédit ne compense pas l'avantage de flexibilité accordé aux producteurs Bio, il faut augmenter le seuil $l$. Dès que $b_{o} S^{\prime}[l]<a_{o}^{\prime}[l]$, l'effet de discrédit sur la filière Bio l'emporte et augmenter $l$ diminue le surplus total. Dans ces conditions, $l^{*}$ est une solution intérieure au problème de maximisation, telle que $l^{*} \neq 0$ si et seulement si $b_{o} S^{\prime}[l=0]>a_{o}{ }^{\prime}[l=0]$. En effet, si $b_{o} S^{\prime}[l=0]<a_{o}^{\prime}[l=0]$, alors il n'est pas possible d'augmenter $l$ sans détériorer le surplus.

Avec la spécification retenue, $a_{o}^{\prime}[l=0]=-\bar{a}_{o}$ et $b_{o} S^{\prime}[l=0]=-2 b_{o} \lambda$. Si les valeurs des paramètres sont tels que $2 b_{o} \lambda>\bar{a}_{o}$, alors $l^{*}>0$.

\section{(II) (iii) Démonstration de la caractéristique de $l^{*}$ quant à la répartition des consommateurs entre les deux agricultures}

Pour démontrer que le seuil $l^{*}$ qui maximise le surplus total, minimise dans le même temps $\tilde{e}[l]$ (ou encore maximise la part $(1-\tilde{e}[l])$ des consommateurs de produits Bio dans l'ensemble des consommateurs), il suffit de comparer les fonctions $\tilde{e}[l]$ et $S T[l]$.

Commençons par étudier la fonction $\tilde{e}[l]$. Elle peut être obtenue en égalisant les expressions (5) et (6) mais également en maximisant le surplus total par rapport à $e$. En effet, conformément à la Figure 1, la valeur de ẽ à l'intersection des deux fonctions de surplus correspond dans le même temps au ẽ qui maximise l'ensemble de ces deux fonctions.

Déterminons ẽ en maximisant la fonction de surplus total.

$$
\frac{d S T}{d \tilde{e}}=0 \Rightarrow F^{2}-2 F G \tilde{e}+G^{2} \tilde{e}^{2}-\left(a_{o}[l]+X \tilde{e}-b_{o} S[l]-Y\right)^{2}=0
$$

On obtient un seul maximum : $\tilde{e}^{*}=\frac{F-\left(a_{o}[l]-b_{o} S[l]-Y\right)}{G+X}$

Lorsqu'on maximise par rapport à $l$ cette fonction $\tilde{e}[l]$, on trouve la CPO suivante :

$$
\frac{d \tilde{e}[l]}{d l}=0 \Rightarrow \frac{-a_{o}^{\prime}[l]+b_{o} S^{\prime}[l]}{G+X}=0
$$


Il vient que la fonction $\tilde{e}[l]$ atteint un extremum pour une valeur de $l^{*}$ telle que $a_{o}^{\prime}[l]=b_{o} S^{\prime}[l]$. L'étude de la CSO montre qu'il s'agit d'un minimum si $a_{o}^{\prime \prime}[l]=0$ puisque $S^{\prime \prime}[l]>0$.

Ainsi, c'est la même valeur de $l$ telle que $a_{o}{ }^{\prime}[l]=b_{o} S^{\prime}[l]$ qui minimise $\tilde{e}[l]$ et qui maximise le surplus total.

En conclusion, maximiser le surplus total revient à minimiser la fonction $\tilde{e}[l]$ et conduit donc à avoir le plus grand nombre possible de consommateurs de produits Bio.

Avec la spécification retenue, il est aisé de vérifier que $\tilde{e}[l]$ est une fonction du second degré et que sa dérivée seconde par rapport à $l$ est positive quelle que soit la valeur des paramètres $\left(\partial^{2} \tilde{e}[l] / \partial l^{2}>0\right)$. On en déduit que la fonction $\tilde{e}[l]$ est une courbe en $\mathrm{U}$ qui atteint donc un seul minimum en $l^{*}=1-\frac{\bar{a}_{o}}{2 b_{o} \lambda}$.

\section{Annexe B : Démonstration de la Proposition 2}

\section{(I) Démonstration de l'existence de $l^{*}$}

La prise en compte du principe du newcomer n'influençant que le type de producteur supportant le coût et non la forme de ce coût -la fonction $S[l]$ garde les mêmes propriétés (continue et deux fois dérivable sur l'intervalle $l \in[0,1]$ )-, la fonction de surplus total $S T[l]$ atteint, pour les mêmes raisons évoquées dans l'Annexe A(I), toujours au moins 1 maximum en $l$ sur l'intervalle $[0,1]$ et ce, quelle que soit la valeur des paramètres qui assurent la coexistence des agricultures.

\section{(II) (i) Démonstration de la détermination de $l^{*}$ en tant que solution en coin au problème de maximisation}

Conservons les mêmes hypothèses sur les fonctions de demande et reprenons la simplification de l'expression du surplus réalisée dans l'Annexe A(II)(i) en l'adaptant au principe du newcomer. Nous écrivons alors :

$$
S T^{N C}[l]=n \int_{e=0}^{\tilde{e}[l]}\left(F-G e-b_{g m} S[l]\right)^{2} \mathrm{~d} e+n \int_{e=\tilde{e}[l]}^{1}\left(a_{o}[l]+X e-Y\right)^{2} \mathrm{~d} e
$$

avec $F-G e-b_{g m} S[l]>0$ (condition de positivité de la demande OGM) et $a_{o}[l]-Y>0$ (condition de positivité de la demande Bio).

La maximisation du surplus par rapport à $l$ conduit à poser la CPO suivante :

$$
\begin{gathered}
\frac{d S T^{N C}[l]}{d l}=0 \\
\Rightarrow \underbrace{(1-\tilde{e})\left(2 a_{o}[l]+X \tilde{e}+X-2 Y\right)\left(a_{o}^{\prime}[l]\right)}_{\max _{l} \text { Surplus Bio }}+\underbrace{\tilde{e}\left(2 F-G \tilde{e}-2 b_{g m} S[l]\right)\left(-b_{g} m S^{\prime}[l]\right)}_{\max _{l} \text { Surplus OGM }}=0
\end{gathered}
$$

Les surplus OGM et Bio sont maximisés de manière distincte. Il n'est pas possible de déterminer un extremum de façon évidente comme nous le faisions sans le principe du newcomer. Ici l'égalité entre les gains liés à la flexibilité pour le producteur OGM et les pertes liées au discrédit sur la filière Bio $\left(a_{g m}^{\prime}[l]=b_{o} S^{\prime}[l]\right)$ n'est pas une solution au problème de maximisation. 
Il convient d'étudier la fonction $\tilde{e}[l]$ ainsi que chacun des surplus de façon distincte.

Déterminons ẽ en procédant de la même manière que dans l'Annexe A(II)(iii) : en maximisant la fonction de surplus total.

$$
\frac{d S T^{N C}}{d \tilde{e}}=0 \Rightarrow\left(-F+G \tilde{e}+b_{g m} S[l]\right)^{2}-\left(a_{o}[l]+X \tilde{e}-Y\right)^{2}=0
$$

On obtient un seul maximum : $\tilde{e}^{*}=\frac{\left.F-b_{g m} S[l]-a_{o}[l]+Y\right)}{G+X}$

Lorsqu'on maximise par rapport à $l$ cette fonction $\tilde{e}[l]$, on obtient une dérivée première strictement positive car $a_{o}^{\prime}[l]<0$ et $b_{g m} S^{\prime}[l]<0$ :

$$
\frac{d \tilde{e}[l]}{d l} \Rightarrow \frac{-a_{o}^{\prime}[l]-b_{g m} S^{\prime}[l]}{G+X}>0
$$

On en déduit que la fonction $\tilde{e}[l]$ est strictement croissante sur l'intervalle $l \in[0,1]$. Autrement dit, la fonction $\tilde{e}[l]$ atteint un minimum pour une valeur de $l^{*}=0$.

Étudions à présent le seuil $l^{*}$ qui maximise le surplus Bio.

$$
\frac{d \operatorname{Surplus}_{o}[l]}{d l}=(1-\tilde{e})\left(2 a_{o}[l]+X \tilde{e}+X-2 Y\right)\left(a_{o}^{\prime}[l]\right)
$$

Lorsqu'on étudie le surplus Bio sans tenir compte de $\tilde{e}$, il apparaît clairement que le surplus Bio est strictement décroissant de $l$ puisque $a_{o}^{\prime}[l]<0$. Si l'on étudie comment le surplus Bio varie en fonction de $\tilde{e}$, il en ressort qu'il est strictement décroissant (en effet plus le nombre de consommateurs Bio est élevé et plus le surplus Bio l'est également). Comme $\tilde{e}$ est minimum si $l^{*}=0$, on en conclut que le surplus Bio est maximum dès lors que $l^{*}=0$.

Étudions maintenant le seuil $l^{*}$ qui maximise le surplus OGM.

$$
\frac{d \text { Surplus }_{g m}[l]}{d l}=\tilde{e}\left(2 F-G \tilde{e}-2 b_{g m} S[l]\right)\left(-b_{g} m S^{\prime}[l]\right)
$$

Il en ressort que le surplus OGM est strictement croissant de $l$ puisque $S^{\prime}[l]<0$ et $\tilde{e}$ est strictement croissant de $l$. On en conclut que le surplus OGM est maximum dès lors que $l^{*}=1$.

A partir de l'étude de ces deux surplus, nous pouvons enfin déterminer le $l^{*}$ qui maximise le surplus total. Comme le surplus total équivaut à la somme du surplus Bio et du surplus OGM, nous en déduisons que le surplus dont le poids est le plus important sera celui qui déterminera la valeur de $l^{*}$. Compte tenu des hypothèses faites sur les fonctions de demande $\left(a_{o}[e, l]>a_{g m}[e]\right.$ et $\left.b_{o}<b_{g m}\right)$, on en déduit que le surplus Bio est déterminant dans le surplus total et donc que $l^{*}=0$.

\section{(II) (ii) Démonstration de la caractéristique de $l^{*}$ quant à la répartition des consommateurs entre les deux agricultures}

Dans l'Annexe B(II)(i), nous avons montré que c'est la même valeur de $l$ telle que $l^{*}=0$ qui minimise $\tilde{e}[l]$ et qui maximise le surplus Bio et de fait le surplus total.

En conclusion avec ou sans le principe du newcomer, maximiser le surplus total revient à minimiser la fonction $\tilde{e}[l]$ et conduit donc à avoir le plus grand nombre possible de consommateurs de produits Bio. 


\section{Bibliographie}

Balineau, G., et Dufeu, I. (2010), "Are Fair Trade Goods Credence Goods? A New Proposal, with French Illustration", Journal of Business Ethics, 92, 331-345.

Batte, M. T., Hooker, N. H., Haab, T. C., et Beaverson J. (2007), "Putting their Money where their Mouths are : Consumer Willingness to Pay for Multi-Ingredient, Processed Organic Food Products", Food Policy, 32, 145-159.

Beckmann, V., Soregaroli, C., et Wesseler, J. (2010), "Ex ante regulation and Ex post liability under uncertainty and irreversibility : Governing the coexistence of GM crops", Economics, http ://www.economics.ejournal.org/economics/journalarticles/2010-9.

Beckmann, V., Soregaroli, C., et Wesseler, J. (2006), "Coexistence rules and regulations in the European Union", American Journal of Economics, 88(5), 1193-1199.

Ceddia, M.G., Bartlett, M., De Lucia, C. et Perrings, C. (2011), "On the regulation of spatial externalities : Coexistence between GM and conventional crops in the EU and the 'newcomer principle'", Australian Journal of Agricultural and Resource Economics, $55,126-143$.

Crespi, J. M., et Marette, S. (2003), “Does Contain' vs. 'Does Not Contain' : Does it Matter which GMO Label is Used?", European Journal of Law and Economics, 16, 327-344.

Demont, M. et Devos, Y. (2008), "Regulating coexistence of GM and non-GM crops without jeopardizing economic incentives", Trends in Biotechnologies, 26, 353-358.

Demont, M., Dillen, K., Daems, W., Sausse, C., Tollens, E. et Mathijs, E. (2009), "On the proportionality of EU spatial ex ante coexistence regulations", Food Policy, 34, 508-518.

Desquilbet, M. et Bullock, D. S. (2010), "On the proportionality of EU spatial ex ante coexistence regulations: A comment", Food Policy, 35, 87-90.

Darby, M., et Karni, E. (1973), "Free Competition and the Optimal Level of Fraud", Journal of Law and Economics, 16(1), 67-88.

Fulton, M., et Giannakas, K. (2004), "Inserting GM Products into the Food Chain : The Market and Welfare Effects of the Different Labeling and Regulatory Regimes", American Journal of Agricultural Economics, 86(1), 42-60.

Giannakas, K. (2002), "Information Asymmetries and Consumption Decisions in Organic Food Product Markets", Canadian Journal of Agricultural Economics, 50(1), 35-50.

Giannakas, K., et Yiannaka, A. (2006), "Agricultural Biotechnology and Organic Agriculture : National Organic Standards and Labeling of GM Products", AgBioForum, 9(2), 84-93.

Gray, E., Ancev, T. et Drynan, R. (2011), "Coexistence of GM and non-GM crops with endogenously determined separation", Ecological Economics, 70, 2488-2493.

Huygen, I., Veeman, M. et Lerohl, M. (2004), "Cost Implications of Alternative GM Tolerance Levels : Non-Genetically Modified Wheat in Western Canada", AgBioForum, 6(4), 169-177.

Lupton, S. (2005), "Shared Quality Uncertainty and the Introduction of Indeterminate Goods", Cambridge Journal of Economics, 29, 399-345.

Lusk, J. L., et Briggeman, B. C. (2009), "Food Values", American Journal of Agricultural Economics, 91(1), 184-196. 
Nelson, P. (1970), "Information and consumer behavior", Journal of Political Economy, 78(2), 311-329.

Ngo, M. A. (2011), "Le droit de la responsabilité en matière d'OGM : de la prévention à la réparation", Droit de l'Environnement, 196, 352-355.

Nimon, W., et Beghin, J. (1999), "Are Eco-Labels Valuable" ? Evidence from the Apparel Industry", Journal of Agricultural Economics, 81(6), 801-811.

Noussair, C., Robin, S., et Ruffieux, B. (2004), "Do Consumers Really Refuse to Buy Genetically Modified Food?", Economic Journal, 114, 102-120.

Runge, C. F., et Jackson, L. A. (2000), "Labelling, Trade and Genetically Modified Organisms", Journal of World Trade, 34, 111-122.

Sawyer, E.N., Kerr, W. A., et Hobbs, J. E. (2007), "Consumer Preferences and the International Harmonization of Organic Standards", Food Policy, 33, 607-615. 